\title{
Embeddings of Graphs in Euclidean Spaces
}

\author{
J. Reiterman, V. Rödl, and E. Šin̆ajová \\ Department of Mathematics FJFI, Technical University of Prague, Husova 5, \\ 11000 Prague 1, Czechoslovakia
}

\begin{abstract}
The dimension of a graph $G=(V, E)$ is the minimum number $d$ such that there exists a representation $x \rightarrow \bar{x} \in R^{d}(x \in V)$ and a threshold $t$ such that $x y \in E$ iff $\bar{x} \bar{y} \geq t$. We prove that $\mathrm{d}(G) \leq n-\chi(G)$ and $\mathrm{d}(G) \leq n-\sqrt{n}$ where $n=|V|$ and $\chi(G)$ is the chromatic number of $G$; we present upper bounds for the dimension of graphs with a large girth and we show that the complement of a forest can be represented by unit vectors in $R^{6}$. We prove that $\mathrm{d}(G) \geq \frac{1}{15} n$ for most graphs and that there are 3-regular graphs with $\mathrm{d}(G) \geq c \log n / \log \log n$.
\end{abstract}

\section{Introduction}

We consider the geometric dimension of graphs introduced in [7]. Let $G=(V, E)$ be a finite graph without loops and multiple edges. The dimension $\mathrm{d}(G)$ of $G$ is the minimum number $d$ such that $G$ admits a representation in $R^{d}$; a representation consists of an assignment $x \rightarrow \bar{x} \in R^{d}(x \in V)$ and a threshold $t \in R$ such that for every couple $x, y \in V, x \neq y$,

$$
x y \in E \quad \text { iff } \quad \bar{x} \bar{y} \geq t,
$$

where $\bar{x} \bar{y}$ is the scalar product of vectors $\bar{x}, \bar{y}$.

In [7] we also considered a related notion-the spherical dimension $\operatorname{sd}(G)$ of a graph $G$, which is defined analogously but with an additional condition $\|\bar{x}\|=1$ for all $x \in V$. Clearly, $\operatorname{sd}(G) \geq \mathrm{d}(G)$ for every graph $G$.

The notion of spherical dimension is closely related to that of sphericity which was introduced in [4]. The sphericity is defined as the minimum number $d$ such that there exists an assignment $x \rightarrow \bar{x} \in R^{d}$ and a threshold $\rho \geq 0$ such that

$$
x y \in E \quad \text { iff } \quad\|\bar{x}-\bar{y}\| \leq \rho .
$$


In [7] it is noted that

$$
\operatorname{sd}(G)-1 \leq \operatorname{sph}(G) \leq \operatorname{sd}(G)
$$

for every graph $G$.

Let us list some bounds for $\mathrm{d}(G), \operatorname{sd}(G)$, and $\operatorname{sph}(G)$ :

(1) $\mathrm{d}(G) \leq n-\alpha(G)(n=|V|, \alpha(G)$ is the size of a maximal independent set in $G$ ).

(2) $\operatorname{sph}(G) \leq n-\omega(G)$ (where $\omega(G)$ is the size of a maximal clique in $G$ ).

(3) $\operatorname{sd}(G) \leq c d \log (d)$ if the complement $\bar{G}$ of $G$ has maximum degree $\leq d$.

(4) $\mathrm{d}(T) \leq 3$ if $T$ is a tree.

(5) $\operatorname{sph}(T) \leq \frac{1}{2}(k+1) \log _{k}|T|$ if $T$ is a tree with degree $\leq k$.

(6) $\operatorname{sph}(T) \leq 108 \log |T|$ if $T$ is a tree.

(7) $\operatorname{sph}(F) \leq 8[\log |F|\rceil$ if $F$ is a forest.

(8) $c_{1} n / \log n \leq \operatorname{sph}\left(Q_{n}\right) \leq c_{2} n / \log n$ where $Q_{n}$ is the graph of the $n$ dimensional cube.

(9) $\operatorname{sd}(G) \leq c d^{2} \log n$ if $G$ has maximum degree $\leq d$.

(10) $\operatorname{sph}\left(G_{1}+G_{2}+\cdots+G_{m}\right) \leq 2(n-1)$ where $n=\max V\left(G_{1}\right)$.

(11) $\mathrm{d}\left(\bigcup_{i} G_{i}\right) \leq \operatorname{maxd}\left(G_{i}\right)+1$ where the $G_{i}$ 's are disjoint.

(12) $\mathrm{d}\left(G \cup K_{A}\right) \leq \mathrm{d}(G)+1$ where $A \subset V$ and $K_{A}$ is a clique on $A$.

(13) $\operatorname{sph}(G)<12(2 c-1)^{2} \log |G|$ for $\lambda_{\min } \geq-c \quad(c \geq 2)$ and $|G|>$ $\left[12(2 c-1)^{2} \log |G|\right]^{2}$, where $\lambda_{\min }$ is the minimal eigenvalue of $G$.

(14) $\operatorname{sph}(L(G))<108 \log m$ where $m=|E|$ and $m>(108 \log m)^{2}$.

(15) $\operatorname{sph}(G) \geq \log \alpha(G) /(\log (2 r(G)+1))$ where $\mathrm{r}(G)$ is the radius of $G$.

For these and related further results see [1]-[4] and [7].

The aim of this paper is to present some other upper and lower results for $\mathrm{d}(G)$. In Section 1 we prove that $\mathrm{d}(G) \leq n-\chi(G)$ where $\chi(G)$ is the chromatic number of $G$ which, together with (1) above, yields $\mathrm{d}(G) \leq n-\sqrt{n}$. In Section 2 we consider the dimension of forests and graphs with a large girth. In Section 3 we deal with lower bounds for $\mathrm{d}(G)$. We prove that most of the graphs on $n$ vertices have $\mathrm{d}(G) \geq n / 15$ for sufficiently large $n$. We also prove that, contrary to (3) above, there are graphs $G_{n}$ with maximum degree $\leq 3$ but with $\mathrm{d}\left(G_{n}\right)>$ $\log n / 18 \log \log n$.

\section{General Upper Bounds}

1.1. Theorem. For every graph on $n$ vertices,

$$
\mathrm{d}(G) \leq n-\chi(G)
$$

Proof. 1. The set $V$ of vertices of $G=(V, E)$ with $\chi(G)=\chi$ can be written as

$$
V=K \cup A_{k+1} \cup A_{k+2} \cup \cdots \cup A_{x},
$$


where $K$ is a clique of size $k$, each $A_{i}$ has size $\geq 2$, and $A_{i}$ is a maximal independent set in the induced subgraph $G_{i}$ on $K \cup A_{k+1} \cup \cdots \cup A_{i}, k+1 \leq i \leq \chi$.

Indeed, let $A_{1} \cup \cdots \cup A_{\chi}$ be the decomposition of $V$ corresponding to a coloring of $G$ such that $\left|A_{1}\right| \leq\left|A_{2}\right| \leq \cdots \leq\left|A_{\chi}\right|$. If $A_{\chi}$ is not maximal independent in $G_{x}$, we move suitable vertices of $A_{1} \cup \cdots \cup A_{x-1}$ to $A_{x}$ to make it such. Then we proceed in the same way with $A_{x-1}, A_{x-2}, \ldots, A_{1}$. Thus if $k+1$ is the least integer with $\left|A_{k+1}\right| \geq 2$ then $K=A_{1} \cup \cdots \cup A_{k}$ is a clique of size $k$.

2. Let $\left|A_{k+1}\right|=d+1$. Then $G_{k+1}$ can be represented in $R^{d}$ with a threshold $t<0$. Indeed, consider the vertices $\bar{z}_{0}, \ldots, \bar{z}_{d}$ of the regular simplex in $R^{d}$ centered at the origin with

$$
\bar{z}_{i} \bar{z}_{j}=-1 \quad(i \neq j), \quad \bar{z}_{i}^{2}=d
$$

Let $K=\left\{x_{1}, \ldots, x_{k}\right\}, A_{k+1}=\left\{u_{0}, \ldots, u_{d}\right\}$. Put

$$
\bar{x}_{i}=\sum_{r \in I_{1}} \bar{z}_{r} \quad(i=1, \ldots, k),
$$

where

$$
I_{1}=\left\{r \mid x, u_{r} \in E\right\}
$$

and

$$
\bar{u}_{j}=2 d^{2} \bar{z}_{j} \quad(j=0, \ldots, d)
$$

We have:

(a) $\bar{x}_{i} \bar{x}_{y}=\sum\left\{\bar{z}_{r} \bar{z}_{s} \mid r \in I_{1}, s \in I_{1}\right\} \geq-d^{2}$.

(b) $\bar{u}_{i} \bar{u}_{i}=-4 d^{4}<-d^{2}$ for $i \neq j$.

(c) If $x_{i} u_{j} \in E$ then

$$
\begin{aligned}
\bar{x}_{i} \bar{u}_{J} & =2 d^{2} \sum\left\{\bar{z}_{r} \bar{z}_{j} \mid r \in I_{i}\right\} \\
& =2 d^{3}+2 d^{2} \sum\left\{\bar{z}_{r} \bar{z}_{j} \mid r \in I_{r}-\{j\}\right\} \geq 2 d^{3}-2 d^{3}=0 .
\end{aligned}
$$

(d) If $x_{i} u_{j} \notin E$ then $\bar{x}_{i} \bar{u}_{j}=-2 d^{2}\left|I_{i}\right|<-d^{2}$ for $I_{i} \neq \varnothing$ because of the maximality of $A_{k+1}$.

Thus vectors $\bar{x}_{i}, \tilde{u}_{j}$ form a representation of $G_{k+1}$ in $R^{d}$ with the threshold $t=-d^{2}$.

3. Suppose $G_{h}$ can be represented in $R^{d}$ with a threshold $t<0$. Then $G_{h+1}$ can be represented in $R^{d+5}$, where $s+1=\left|A_{b+1}\right|$, with a negative threshold. Indeed, let $x_{1}, \ldots, x_{m}$ be vertices of $G_{h}$ and $u_{0}, \ldots, u_{\text {s }}$ vertices of $A_{h+1}$. Let $\bar{x}_{1}, \ldots, \bar{x}_{m}$ form the representation of $G_{h}$ in $R^{d}$ with a threshold $t<0$. Thus there is $t_{1}<t$ such that

$$
\bar{x}_{i} \bar{x}_{j} \geq t \quad \text { if } x_{i} x_{j} \in E \text { and } \quad \bar{x}_{i} \bar{x}_{j} \leq t_{1} \quad \text { if } x_{i} x_{j} \notin E .
$$

Again, choose vectors $\bar{z}_{0}, \ldots, \bar{z}_{\mathrm{s}}$ in $R^{s}$ with

$$
\bar{z}_{i} \bar{z}_{j}=-1 \quad \text { for } \quad i \neq j, \quad \bar{z}_{i}^{2}=s .
$$

Put

$$
\begin{aligned}
& \tilde{x}_{i}=\left(\bar{x}_{i}, \varepsilon \sum_{r \in I_{t}} z_{r}\right) \in R^{d+s}, \quad(i=1, \ldots, m), \\
& \tilde{u}_{j}=\left(\overline{0}, \alpha \bar{z}_{j}\right) \in R^{d+s} \quad(j=0, \ldots, s),
\end{aligned}
$$


where $I_{i}$ is defined as in 2 above and $\alpha>0, \varepsilon>0$ will be specified later. Then we have:

(a) If $x_{i} x_{j} \in E$ then

$$
\tilde{x}_{i} \tilde{x}_{j}=\bar{x}_{i} \bar{x}_{j}+\varepsilon^{2} \sum\left\{\bar{z}_{r} \bar{z}_{u} \mid r \in I_{i}, u \in I_{j}\right\} \geq t-\varepsilon^{2} s^{2} .
$$

(b) If $x_{i} x_{j} E$ then

$$
\tilde{x}_{i} \tilde{x}_{j} \leq t_{1}+\varepsilon^{2} \sum_{r=0}^{s} \bar{z}_{r}^{2} \leq \varepsilon^{2} s(s+1)+t_{1}
$$

(c) If $x_{i} u_{j} \in E$ then

$$
\begin{aligned}
\tilde{x}_{i} \tilde{u}_{j} & =\alpha \varepsilon \sum\left\{\bar{z}_{r}, \bar{z}_{j} \mid r \in I_{i}\right\} \\
& =\alpha \varepsilon \bar{z}_{j}^{2}+\alpha \varepsilon \sum\left\{\bar{z}_{r} \bar{z}_{j} \mid r \in I_{i}-\{j\}\right\} \geq \alpha \varepsilon s-\alpha \varepsilon s=0 .
\end{aligned}
$$

(d) If $x_{i} u_{j} \notin E$ then

$$
\tilde{x}_{i} \tilde{u}_{j}=-\alpha \varepsilon\left|I_{i}\right| \leq-\alpha \varepsilon
$$

because the inclusion maximality of $A_{h+1}$ in $G_{h+1}$ forces $\left|I_{i}\right|>0$.

(e) $\tilde{u}_{i} \tilde{u}_{j}=-\alpha^{2}$ for $i \neq j$.

We are going to show that $\alpha, \varepsilon$ can be chosen such that vectors $\tilde{x}_{i}, \tilde{u}_{j}$ form a representation of $G_{h+1}$ with any threshold $t^{\prime}$ where $t_{1}<t^{\prime}<t<0$. Indeed, first choose $\varepsilon>0$ sufficiently small such that

$$
\begin{aligned}
t-\varepsilon^{2} s^{2}>t^{\prime} & (\text { see }(\text { a })), \\
t_{1}+\varepsilon^{2} s(s+1)<t^{\prime} & (\text { see (b)). }
\end{aligned}
$$

Then choose $\alpha$ sufficiently large such that

$$
\begin{array}{ll}
-\alpha \varepsilon<t^{\prime} & (\operatorname{see}(\mathrm{d})), \\
-\alpha^{2}<t^{\prime} & (\operatorname{see}(\mathrm{e})) .
\end{array}
$$

4. It follows from 2 and 3 above that by induction $G=G_{x}$ can be represented in $R^{d}$ where

$$
\begin{aligned}
d & =\left(\left|A_{k+1}\right|-1\right)+\left(\left|A_{k+2}\right|-1\right)+\cdots+\left(\left|A_{\chi}\right|-1\right) \\
& =|V-K|-(\chi-k)=n-\chi .
\end{aligned}
$$

This concludes the proof of the theorem.

1.2. Corollary. For every graph $G$ on $n$ vertices,

$$
\mathrm{d}(G) \leq n-\sqrt{n}
$$


Proof. In [6] the threshold dimension $\theta(G)$ of a graph $G$ is introduced and shown to satisfy $\theta(G) \leq n-\alpha(G)$ where $\alpha(G)$ is the size of a maximal independent set in $G$. Following [7], $\mathrm{d}(G) \leq \theta(G)$ for every graph $G$. Thus $\mathrm{d}(G) \leq$ $n-\alpha(G)$. As $\alpha(G) \cdot \chi(G) \geq n$, we have $\max (\alpha(G), \chi(G)) \geq \sqrt{n}$, hence

$$
\mathrm{d}(G) \leq \min (n-\alpha(G), n-\chi(G)) \leq n-\sqrt{n} .
$$

\section{Upper Bounds for Graphs Without Cycles}

2.1. Proposition. If $G$ is a forest then $\mathrm{d}(G) \leq 3$; the representation can be chosen to have a positive threshold.

Proof. This is proved in [7] for $G$ a tree; in more detail, for every $\varepsilon>0$, a tree $G=(V, E)$ can be represented in $R^{3}$ with the threshold $t=1$ such that vectors $\bar{x}$ representing vertices $x \in V$ satisfy $1<\|\bar{x}\|<1+\varepsilon$. Then $\operatorname{diam}\{\bar{x} \mid x \in V\} \rightarrow 0$. Thus, as the tree $G$ is connected, its representation can be found to be placed in an arbitrarily small ball with center on the unit sphere $S$ in $R^{3}$. Now, if $G$ is a forest with components $G_{1}, \ldots, G_{m}$, then choose distinct points $c_{1}, \ldots, c_{m}$ and balls $B_{i}$ with centers $c_{i}$, each with a radius $r$. Represent $G_{i}$ in $B_{i}$; if $r$ is small enough, this yields a representation of $G$ in $R^{3}$ with the threshold $t=1$.

2.2. Proposition. If $G$ is a forest that does not contain the tree $T_{0}$ below as an induced subgraph then $\mathrm{d}(G) \leq 2$ where the representation in $R^{2}$ can be chosen to have a positive threshold $t$.

Proof. In fact, this is proved in [7] for $G=(V, E)$ with $t^{\prime}=1-\varepsilon(0<\varepsilon<1)$ where $\varepsilon$ can be arbitrarily small, and vectors $\bar{x} \in R^{2}$.representing vertices of $V$ satisfy $1 \geq\|\bar{x}\| \geq 1-\varepsilon$. Thus the representation can be constructed with $\operatorname{diam}\{\bar{x} \mid x \in$ $V\}$ arbitrarily small. Using the same argument as in the preceding proposition, this can be used to represent any forest without induced $T_{0}$ in $R^{2}$ with a positive threshold (Fig. 1).

2.3. Proposition. A graph $G$ on $n$ vertices can be represented in $R^{d}$ where:

I. $d \leq \frac{2}{7} n+1$ if $G$ has a girth at least 7 ,

II. $d \leq \frac{1}{3} n+\frac{2}{3}$ if $G$ has a girth at least 6 ,

III. $d \leq \frac{2}{5} n+\frac{3}{5}$ if $G$ has a girth at least 5 .

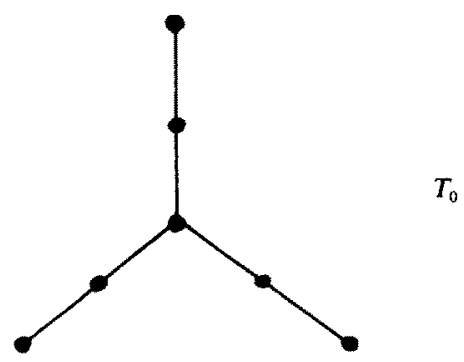

Fig. 1 
Remark. Proposition 2.3 yields linear upper bounds for $\mathrm{d}(G)$ for $G$ without short cycles, which, however, can be further improved (to appear in a subsequent paper). Using a probabilistic approach we can, for example, show an upper bound of the form $c_{1} \rho(\log n)^{c_{2}}$ for any $C_{4}$-free graph with edge density $\rho$. As it is well known that $\rho \leq \sqrt{n}$ for any $C_{4}$-free graph, this clearly improves Proposition 2.3 for $n \geq n\left(c_{1}, c_{2}\right)$. To prove Proposition 2.3 we need the following.

Lemma. Let a graph $G=(V, E)$ contain a set $C$ of vertices spanning one of the graphs $C_{5}, C_{6}, C_{7}, C_{8}, P_{7}$ such that each vertex in $V-C$ is adjacent to at most one of the vertices of $C$. Let the induced subgraph $G^{\prime}$ of $G$ on $V-C$ be represented in $R^{d}$ with a positive threshold. Then $G$ can be represented in $R^{d+2}$ with a positive threshold.

Proof. Let $C=\left\{v_{0}, \ldots, v_{r-1}\right\}$ span $C_{r}, 5 \leq r \leq 8$. Put

$$
\bar{z}_{i}=\left(\cos \frac{2 \pi i}{r}, \sin \frac{2 \pi i}{r}\right) \quad(i=0, \ldots, r-1) .
$$

Then

$$
\begin{array}{ll}
\bar{z}_{i}^{2}=1, & \bar{z}_{i} \bar{z}_{i+1}=\cos \frac{2 \pi}{r} \geq \cos \frac{2 \pi}{5}>0 . \\
\bar{z}_{i} \bar{z}_{j} \leq 0 & \text { for } \quad i \neq j \pm 1 \bmod r, \quad i \neq j .
\end{array}
$$

Let $x \rightarrow \bar{x} \in R^{d}$ be a representation of $G^{\prime}$ in $R^{d}$ with a positive threshold $t$, without loss of generality, $t=1$. Then, if $\alpha>C$ is sufficiently small, vectors

$$
\begin{aligned}
& \tilde{x}=\left(\bar{x}, \alpha \bar{z}_{i}\right) \in R^{d+2} \quad\left(x \in V-C, v_{i} x \in E\right), \\
& \tilde{x}=(\bar{x}, \overline{0}) \in R^{d+2} \quad\left(x \in V-C, v_{i} x \notin E \text { for no } i\right)
\end{aligned}
$$

form a representation of $G^{\prime}$ in $R^{d+2}$ with a threshold $t^{\prime}$, where

$$
\cos \frac{2 \pi}{r}<t^{\prime}<1
$$

Put

$$
\tilde{v}_{i}=\left(\overline{0}, \bar{z}_{i} / \alpha\right)
$$

Then, for sufficiently small $\alpha>0$,

$$
\begin{aligned}
\tilde{v}_{i} \tilde{v}_{i+1} & =\frac{1}{\alpha^{2}} \cos \frac{2 \pi}{r}>1, \\
\tilde{v}_{i} \tilde{v}_{j} & \leq 0 \quad \text { if } \quad i \neq j \pm 1 \bmod r, \quad i \neq j, \\
\tilde{x} \tilde{v}_{i} & =1>t^{\prime} \quad \text { if } \quad x \in V-C \text { is adjacent to } v_{i}, \\
\tilde{x} \tilde{v}_{j} & \leq \cos \frac{2 \pi}{r}<t^{\prime} \quad \text { if } \quad x \in V-C \text { is not adjacent to } v_{j} .
\end{aligned}
$$


This proves that vectors $\tilde{x}(x \in V-C)$ and $\tilde{v}_{i}(i=0, \ldots, r-1)$ form a representation of $G$ in $R^{d+2}$ with the threshold $t^{\prime}$.

If $C$ spans $P_{7}$, add a new vertex $x_{0}$ to $G$ to be adjacent just to an endpoint of $P_{7}$; the resulting graph $G^{\prime}$ contains a copy of $C_{8}$ on $C \cup\left\{x_{0}\right\}$ and hence $G^{\prime}$ can be represented in $R^{d+2}$ with a positive threshold by the preceding part of the proof.

Proof of Proposition 2.3. We shall prove by induction on $n$ that $G$ has a representation in $R^{d}$ with a positive threshold where $d$ is bounded by $\frac{2}{7} n+1$ or $\frac{1}{3} n+\frac{2}{3}$ or $\frac{2}{5} n+\frac{3}{5}$ in cases I, II, and III, respectively. This is certainly true if $n \leq 3$. Let $n>3$.

(a) Let $G$ be a forest. Then $\mathrm{d}(G) \leq 3 \leq \frac{2}{7} n+1 \leq \frac{1}{3} n+\frac{2}{3} \leq \frac{2}{5} n+\frac{3}{5}$ if $n \geq 7$, see 2.1 above. If $4 \leq n<7$ then $\mathrm{d}(G) \leq 2 \leq \frac{1}{3} n+\frac{2}{3} \leq \frac{2}{7} n+1 \leq \frac{2}{5} n+\frac{3}{5}$ by 2.2 .

(b) Let $G=(V, E)$ not be a forest. Let $D$ be the shortest cycle in $G$ of length $k$. If $k>8$, choose $C \subset D$ spanning $P_{7}$; if $k \leq 8$, let $C=D$. Due to the minimality of $D$, every vertex of the induced subgraph $G^{\prime}$ on $V-C$ is adjacent to at most one vertex of $C$. If $k \geq 7$ or $k=6$ or $k=5$ then $G^{\prime}$ has $\leq n-7$ or $n-6$ or $n-5$ vertices, respectively. By the induction assumption, $G$ can be represented in $R^{d^{\prime}}$ where

$$
d^{\prime} \leq 2(n-7) / 7+1
$$

or

$$
d^{\prime} \leq(n-6) / 3+\frac{2}{3}
$$

or

$$
d^{\prime} \leq 2(n-5) / 5+\frac{3}{5},
$$

respectively, with a positive threshold. By the lemma, $G$ can be represented in $R^{d}$ with a positive threshold where $d=d^{\prime}+2$, hence $d \leq \frac{2}{7} n+1$, or $d \leq \frac{1}{3} n+\frac{2}{3}$ or $d \leq \frac{2}{5} n+\frac{3}{5}$, respectively.

2.4. The following is an essential improvement of a result of Frankl and Maehara [2] who proved

$$
\operatorname{sd}(G) \leq 8 \log n
$$

if $\bar{G}$ is a forest. Let us remark that $\operatorname{sd}(G)$ is unbounded for trees. It was proved in [7] that

$$
\operatorname{sd}\left(B_{n}\right) \geq c \log n / \log \log n
$$

where $B_{n}$ is the "complete binary" tree on $n=2^{s}-1$ vertices with $s$ levels.

Proposition. If the complement $\bar{G}$ of $G$ is a forest then

$$
\operatorname{sd}(G) \leq 6
$$


Proof. Let $G=(V, E)$ and $\bar{G}$ be a forest. In each component $K_{i}$ of $\bar{G}$, choose a vertex $r_{i}$ to be a root of the tree $K_{i}$. This defines decomposition of each $K_{i}$ into levels. Also, neighbors of each vertex $x$ that is not a root consist of a father and of a family of sons. Let $V_{1}$ be the set of all vertices on odd levels (thus all roots $r_{i}$ are in $V_{1}$ ) and $V_{2}=V-V_{1}$. Then $V_{2}$ can be written as $V_{2}=\left\{x_{1}, \ldots, x_{n}\right\}$ in such a way that sons of each $y \in V_{1}$ form a segment of the form

$$
x_{j}, x_{j+1}, \ldots, x_{k}
$$

Choose reals $\alpha_{i}$,

$$
0<\alpha_{1}<\frac{\pi}{n}<\alpha_{2}<\frac{2 \pi}{n}<\cdots<\frac{(n-1) \pi}{n}<\alpha_{n}<\pi .
$$

Fix a $y \in V_{1}$ with sons as above and with the father $x_{r}$, define a complex polynomial $g_{y}$,

$$
\begin{aligned}
g_{y}(z)= & \left(z-\exp \left(i \frac{\pi(j-1)}{n}\right)\right)\left(z-\exp \left(i \frac{k \pi}{n}\right)\right) \exp \left(-i \frac{\pi(j+k-1)}{2 n}\right) \\
& \times\left(z-\exp \left(i \frac{\pi(r-1)}{n}\right)\right)\left(z-\exp \left(i \frac{\pi r}{n}\right)\right) \exp \left(-i \frac{\pi(2 r-1)}{2 n}\right)
\end{aligned}
$$

We can verify that the coefficients of $g_{y}$ are of the form

$$
g_{y}(z)=c_{2}+c_{1} z+c_{0} z^{2}+\bar{c}_{1} z^{3}+\bar{c}_{2} z^{4}, \quad c_{0} \in R
$$

Put $b_{i}^{*}=2 \operatorname{Re} c_{i}, a_{i}^{*}=2 \operatorname{Im} c_{i}(i=1,2)$, and $d_{0}^{*}=c_{0}$. For a real $\alpha$, define $f_{y}(\alpha)=$ $g_{y}(\exp i \alpha) \cdot \exp (-2 i \alpha)$. Then

$$
\begin{aligned}
f_{y}(\alpha) & =c_{2} e^{-2 i \alpha}+c_{1} e^{-i \alpha}+c_{0}+\bar{c}_{1} e^{i \alpha}+\bar{c}_{2} e^{2 i \alpha} \\
& =d_{0}^{*}+a_{1}^{*} \sin \alpha+b_{1}^{*} \cos \alpha+a_{2}^{*} \sin 2 \alpha+b_{2}^{*} \cos 2 \alpha .
\end{aligned}
$$

Thus $f_{y}$ is real valued, $f_{y}(\alpha)=\left(d_{0}^{*}, a_{1}^{*}, b_{1}^{*}, a_{2}^{*}, b_{2}^{*}\right) \cdot(1, \sin \alpha, \cos \alpha, \sin 2 \alpha$, $\cos 2 \alpha)$. As the only roots of $g_{y}$ are $\exp (i(\pi(j-1) / n)), \exp (i(\pi k / n)), \exp (i(\pi(r-$ $1) / n)), \exp (\mathrm{i}(\pi r / n))$ we see that the only roots of $f_{y}$ are $\pi(j-1) / n, \pi k / n$, $\pi(r-1) / n$, and $\pi r / n$. Hence $f_{y}$ has the same sign at $\alpha_{j}, \alpha_{j+1}, \ldots, \alpha_{k-1}, \alpha_{k}$, and $\alpha_{r}$, and the opposite sign at the other $\alpha_{i}$ 's. Put

$$
\begin{gathered}
\varepsilon=-\operatorname{sgn} f_{y}\left(\alpha_{r}\right) \sqrt{3} / \sqrt{d_{0}^{* 2}+a_{1}^{* 2}+b_{1}^{* 2}+a_{2}^{* 2}+b_{2}^{* 2}} \\
h_{y}=\varepsilon f_{y}, \quad d_{0}^{y}=\varepsilon d_{0}^{*}, \quad a_{i}^{y}=\varepsilon a_{i}^{*}, \quad b_{i}^{y}=\varepsilon b_{i}^{*} \quad(i=1,2) .
\end{gathered}
$$

Then $h_{y}\left(\alpha_{i}\right)<0$ for $j \leq i \leq k$ and for $i=r$ and $h_{y}\left(\alpha_{i}\right)>0$ otherwise. 
Put

$$
\begin{aligned}
& \bar{y}=\left(d_{0}^{y}, a_{1}^{y}, b_{1}^{y}, a_{2}^{y}, b_{2}^{y}, 2\right), \\
& \bar{x}_{i}=\left(1, \sin \alpha_{i}, \cos \alpha_{i}, \sin 2 \alpha_{i}, \cos 2 \alpha_{i},-2\right) .
\end{aligned}
$$

Then

$$
\begin{aligned}
\bar{x}_{i} \bar{y} & =h_{y}\left(\alpha_{i}\right)-4<-4 \quad \text { for } \quad j \leq i \leq k, \quad i=r, \text { i.e., for } x_{i} y \in E, \\
\bar{x}_{i} \bar{y} & =h_{y}\left(\alpha_{1}\right)-4>-4 \text { otherwise, } \\
\bar{x}_{i} \bar{x}_{j} & >-1+4>-4 \text { for all } i, j, \\
\left\|\bar{x}_{i}\right\| & =\|\bar{y}\|=\sqrt{7} .
\end{aligned}
$$

Using the same construction for all $y \in V_{1}$, we also see that, for $y_{1}, y_{2} \in V_{1}$,

$$
\bar{y}_{1} \bar{y}_{2}>-3+4>-4 \text {. }
$$

Thus we have a representation of $G$ in $R^{6}$ by vectors with the same norm $\sqrt{7}$ and with the threshold $t=-4$ which concludes the proof.

\section{Lower Bounds}

3.1. We shall prove that most graphs on $n$ vertices have dimension $\geq n / 15-1$. However, the only graphs of that dimension we explicitly know are those containing $K_{r r}$ for $r>n / 15[7]$.

Theorem. Let $t_{n}$ be the total number of graphs $G$ on $n \geq 38$ vertices. Then at least $(1-1 / n) t_{n}$ of them have

$$
d(G) \geq n / 15-1 \text {. }
$$

Proof. 1. Let $G_{1}, G_{2}, \ldots, G_{t_{n}}$ be a list of all graphs on the set $V=\left\{x_{1}, \ldots, x_{n}\right\}$ of vertices where $\mathrm{d}\left(G_{1}\right) \leq \mathrm{d}\left(\hat{G}_{2}\right) \leq \cdots \leq \mathrm{d}\left(G_{t_{n}}\right)$. Let $r=\left\lfloor t_{n} / n\right\rfloor, d=\mathrm{d}\left(G_{r+1}\right)+1$. Then $G_{1}, \ldots, G_{r}$ can be represented in $R^{d^{n}}$ with the threshold 1; in fact, if $x_{i} \rightarrow \bar{x}_{i} \in R^{d-1}$ is a representation in $R^{d-1}$ with a threshold $t<1$ (or $t \geq 1$ ) then $x_{i} \rightarrow\left(\bar{x}_{i}, \sqrt{1-t}\right)$ or $\left(x_{i} \rightarrow\left(\bar{x}_{i} / t, 0\right)\right.$, resp. $)$ is a representation in $R^{d}$ with the threshold 1. Also, using small changes of coordinates we can modify the representations in such a way that $\bar{x}_{i} \bar{x}_{j}>1$ rather than $\bar{x}_{i} \bar{x}_{j} \geq 1$ if $x_{i} x_{j}$ is an edge, and that vectors $\bar{x}_{1}, \ldots, \bar{x}_{d}$ representing vertices $x_{1}, \ldots, x_{d}$ are linearly independent. Finally, if $\tilde{x}_{i}=\left(\tilde{x}_{i 1}, \ldots, \tilde{x}_{i d}\right) \in R^{d}$ is the vector of coordinates of $x_{i}$ with respect to the orthonormal basis obtained from the base $\bar{x}_{1}, \ldots, \bar{x}_{d}$ by the Gramm-Schmidt orthonormalization process, then $\bar{x}_{i} \bar{x}_{j}=\tilde{x}_{i} \tilde{x}_{j} ;$ moreover, obviously $\tilde{x}_{i, i+1}=$ $\tilde{x}_{i, i+2}=\cdots=\tilde{x}_{i d}=0$ for $i=1, \ldots, d-1$. To summarize our consideration, we have representations $x_{i} \rightarrow \tilde{x}_{i}^{G}$ of graphs $G=G_{1}, \ldots, G_{r}$ in $R^{d}$ such that

$$
\begin{aligned}
\tilde{x}_{i}^{G} \tilde{x}_{j}^{G}>1 & \text { if } x_{i} x_{j} \text { is an edge in } G, \\
\tilde{x}_{i}^{G} \tilde{x}_{j}^{G}<1 & \text { if } x_{i} x_{j} \text { is a nonedge in } G, \\
\tilde{x}_{i j}^{G}=0 & \text { if } \quad 1 \leq i<j \leq d .
\end{aligned}
$$


2. Define polynomials $P_{i j}(1 \leq i<j \leq n)$ in $(n d-(d(d-1) / 2))$ variables $x_{11}$, $x_{21}, x_{22}, x_{31}, x_{32}, x_{33}, \ldots, x_{d 1}, \ldots, x_{d d}, x_{d+1,1}, \ldots, x_{d+1, d}, \ldots, x_{n 1}, \ldots, x_{n d}$ by

$$
P_{i j}\left(x_{u v}\right)_{u, v}=\sum_{k=1}^{d} x_{i k} x_{j k}-1
$$

(where $x_{i k}=0$ if $1 \leq i<k \leq d$ and analogously for $x_{j k}$ ). Then for $\tilde{x}^{G}=\left(\tilde{x}_{w v}^{G}\right)_{u, v} \in R^{N}$ (where $N=n d-(d(d-1) / 2)$ ) we have

$$
\begin{array}{ll}
P_{i j}\left(\tilde{x}^{G}\right)>0 & \text { if } x_{i} x_{j} \text { is an edge in } G, \\
P_{i j}\left(\tilde{x}^{G}\right)<0 & \text { if } x_{i} x_{j} \text { is a nonedge in } G .
\end{array}
$$

3. Following a result of Warren [5], given arbitrary polynomials $P_{1}, \ldots, P_{M}$ on $N$ variables of degree $\leq D$ where $M \geq N$, the total number of sign sequences $\left(\operatorname{sgn} P_{1}(x), \ldots, \operatorname{sgn} P_{M}(x)\right)$ that consist of terms $+1,-1$ does not exceed $(4 e D M / N)^{N}$.

4. Let us apply this estimate to our polynomials $P_{i j}$; as distinct graphs $G \neq G^{\prime}$ induce distinct sign sequences of $P_{i j}$ at $\tilde{x}^{C}$ and $\tilde{x}^{G^{\prime}}$, we have

$$
r \leq(4 e D M / N)^{N}
$$

Here $r=2^{\left(\frac{n}{2}\right)} / n, D=2, M=\left(\begin{array}{l}n \\ 2\end{array}\right)$, and $N=n d-(d(d-1) / 2)$. Hence

$$
\begin{aligned}
r & \leq\left(8 e\left(\begin{array}{l}
n \\
2
\end{array}\right) / N\right)^{N} \leq\left(\frac{4 e}{N / n^{2}}\right)^{N} \\
r^{1 / n^{2}} & \leq\left(\frac{4 e}{y}\right)^{y} \quad \text { where } \quad y=\frac{N}{n^{2}}=\frac{n d-(d(d-1) / 2)}{n^{2}} \in(0,1), \\
\frac{\ln r}{n^{2}} & \leq y^{\ln (4 e / y)} .
\end{aligned}
$$

We have

$$
\frac{\ln r}{n^{2}}=\frac{(\ln 2)\left(\begin{array}{l}
n \\
2
\end{array}\right)-\ln n}{n^{2}}=\frac{\ln 2}{2}-\frac{\ln 2}{2 n}-\frac{\ln n}{n^{2}} \geq \frac{\ln 2}{2}-\frac{1}{85.9}
$$

for $n \geq 38$. The numerical solution of the equation $y \ln (4 e / y)-(\ln 2)) 2+1 / 85.9=$ 0 on $(0,1)$ is $y=1 / 15.26175$, and the function $y \ln (4 e / y)$ is increasing on $(0,1)$. Hence (*) implies $y \geq 1 / 15.262$ for $n \geq 38$. Putting $x=d / n$ we have

$$
y=\frac{d}{n}-\frac{1}{2}\left(\frac{d}{n}\right)^{2}+\frac{1}{2 n}\left(\frac{d}{n}\right)=x-\frac{1}{2} x^{2}+\frac{1}{2 n} x \leq x-\frac{1}{2} x^{2}+\frac{1}{76} x
$$


for $n \geq 38$. Thus

$$
\frac{77}{76} x-\frac{1}{2} x^{2} \geq \frac{1}{15.262}
$$

A numerical solution then gives $x \geq 1 / 14.95>\frac{1}{15}$, hence $d>n / 15$. Then $\mathrm{d}(G)>$ $n / 15-1$ for all $G=G_{r+1}, G_{r+2}, \ldots, G_{t_{n}}$ which concludes the proof.

Remark. The constant $\frac{1}{15}$ can be improved for large $n$ but not to $\frac{1}{14}$ using our method.

3.2. In [7] we proved that if the complement $\bar{G}$ of a graph $G$ has degree bounded by $d$ then $\mathrm{d}(G) \leq 2 d \log (8 d)$, independently of the number of vertices of $G$. We show that this is not the case if $G$ has bounded degree:

Theorem. If $n$ is sufficiently large then there exists a bipartite 3-regular graph $G$ on $2 n$ vertices with

$$
\mathrm{d}(G) \geq \frac{\log n}{18 \log \log n}
$$

To prove the theorem we state two auxiliary lemmas.

Lemma 1. For sufficiently large $n$ there exists a bipartite graph $G=(V, E)$ on $2 n$ vertices such that:

(i) $G$ is 3-regular.

(ii) For every partition $V=A \cup B,|A|=|B|=n$ there exists a matching $M$, $|M|=c n \geq n / 34$ with $e$ having one endpoint in $A$ and another in $B$ for every $e \in M$.

(iii) $G$ does not contain induced cycles of length $\leq 2 t_{o}$ where $t_{0}=\frac{1}{8} \log n$.

Proof. We will proceed by random construction. Let $V=X \cup Y$ and $|X|=|Y|=$ $n$. Let $\pi_{1}, \pi_{2}, \pi_{3}: X \rightarrow Y$ be three random bijections, each taken with probability $1 / n$ ! independently of the choice of the others. Let $\mathbf{G}=(V, E)$ be the "random graph" with edge set $E=\left\{\left\{x, \pi_{i}(x)\right\} ; x \in X, i=1,2,3\right\}$. Thus the probability of a particular graph $G$ equals $\operatorname{pg}(G) /(n !)^{3}$ where $\operatorname{pg}(G)$ denotes the number of ways that $G$ can be written as a union (not necessarily disjoint) of three perfect matchings.

Claim 1. Denote by $p_{n}$ the probability that $G$ is 3-regular. Then

$$
\varliminf_{n \rightarrow \infty} p_{n} \geq 1 / e^{3} .
$$


Proof. The permutation $\pi_{1}$ can be chosen in $n$ ! ways. If $\pi_{1}$ is fixed, then $n$ ! $\left(1-1 / 1 !+1 / 2 ! \cdots+(-1)^{n} / n !\right)$ of permutations $\pi_{2}$ has the property that $\pi_{2}(x) \neq$ $\pi_{1}(x)$ for all $x \in X$, i.e., that edges $x \pi_{i}(x)(x \in X, i=1,2)$ form a 2-regular graph $H$. If $\pi_{2}$ is such a permutation, consider the $n \times n$ matrix $\left(a_{i j}\right)_{i \in X, j \in Y}$ where

$$
\begin{aligned}
& a_{i j}=0 \quad \text { if } i j \text { is an edge of } H, \\
& a_{i j}=1 /(n-2) \text { otherwise. }
\end{aligned}
$$

Then $\sum_{i \in X} a_{i j}=1$ for every $j \in Y$ and $\sum_{j \in Y} a_{i j}=1$ for every $i \in X$.

Hence $\left(a_{i j}\right)$ is a doubly stochastic matrix. By [8] and [9], for the permanent

$$
P=\sum_{\pi \in S_{n}} a_{1 \pi(1)} a_{2 \pi(2)} \cdots a_{n \pi(n)}
$$

of $\left(a_{i j}\right)$ we have $P \geq n ! / n^{n}$. On the other hand, $P=q(1 /(n-2))^{n}$ where $q$ is the number of nonzero summands in $P$. As the nonzero summands clearly correspond to these $\pi$ such that $\pi_{1}, \pi_{2}, \pi$ induce a 3-regular graph, it follows $q \geq$ $n ! /(1-2 / n)^{n}$.

Hence the number of triples $\pi_{1}, \pi_{2}, \pi_{3}$ inducing a 3 -regular graph is $\geq n ! n$ ! $\left(1-1 / 1 !+1 / 2 !-\cdots+(-1)^{n} / n !\right) n !(1-2 / n)^{n}$. Then

$$
p_{n} \geq\left(1-\frac{1}{1 !}+\frac{1}{2 !}-\cdots+\frac{(-1)^{n}}{n !}\right)\left(1-\frac{2}{n}\right)^{n} \rightarrow 1 / e^{3}
$$

which concludes the proof of Claim 1.

Claim 2. Denote by $q_{n}$ the probability that $\mathbf{G}$ satisfies (ii) with $c=\frac{1}{33}$. Then $q_{n} \rightarrow 1$ for $n \rightarrow \infty$.

Proof. Let $V=A \cup B$ be a fixed partition, $|A|=|B|=n, a=|X \cap A|, b=|X-A|$, and $a \geq b$. Then $b=|Y \cap A|, a=|Y-A|$, and $a+b=n$. Set $c=\frac{1}{33}$,

$$
S_{i}^{A}=\left\{(x, y) \mid x \in X \cap Y, y \in Y-A, \pi_{i}(x)=y\right\}
$$

We have

$$
\operatorname{Prob}\left\{\left|S_{i}^{A}\right|<c n\right\} \leq \frac{\sum_{0 \leq j<c n}\left(\begin{array}{c}
a \\
j
\end{array}\right)\left(\begin{array}{c}
b \\
a-j
\end{array}\right)}{\left(\begin{array}{c}
a+b \\
a
\end{array}\right)}
$$

The right-hand side is nonzero only if $b \geq a-j$ for some $j$, i.e., only if $a-c n \leq b$ which yields

$$
a \leq\lfloor(n / 2)(1+c)\rfloor
$$


The right-hand-side of (1) may be further bounded from above by

$$
\frac{\sum_{0 \leq j<c n}\left(\begin{array}{l}
a \\
j
\end{array}\right)^{2}}{\left(\begin{array}{c}
a+b \\
a
\end{array}\right)} \leq \frac{\left(\begin{array}{c}
a \\
\lceil c n\rceil
\end{array}\right)^{2}}{\left(\begin{array}{l}
n \\
a
\end{array}\right)} \leq \frac{\left(\begin{array}{c}
\lfloor(n / 2)(1+c)\rfloor \\
\lceil c n\rceil
\end{array}\right)^{2}}{\left(\begin{array}{c}
n \\
\lfloor(n / 2)(1+c)\rfloor
\end{array}\right)} \leq 2^{-n \varphi(c)+K \log n}
$$

where $\varphi(c)=2 c \log c+\frac{1}{2}(1-c) \log ((1-c) / 2)-\frac{3}{2}(1+c) \log ((1+c) / 2)$ and $K$ is independent of $n$. As $\varphi\left(\frac{1}{33}\right)>\frac{2}{3}$,

$$
\operatorname{Prob}\left\{\left|S_{i}^{A}\right|<c n\right\} \leq 2^{-(2 / 3) n}
$$

for sufficiently large $n$. Then Prob $\left\{\left|S_{i}^{A}\right|<c n \mid i=1,2,3\right\} \leq 2^{-2 n}$. The number of all partitions in question is $\left(\begin{array}{c}2 n \\ n\end{array}\right)$. Thus the probability that $\left|S_{i}^{A}\right|<c n(i=1,2,3)$ for some of them is $\leq\left(\begin{array}{c}2 n \\ n\end{array}\right) 2^{-2 n} \rightarrow 0$.

Claim 3. Denote by $r_{n}$ the probability that $\mathbf{G}$ has the following property: after deleting $\leq \sqrt{n}$ edges from $\mathbf{G}$, the graph " $\mathbf{G}$ minus deleted edges" does not contain any cycle of length $\leq 2 t_{0}$ where $t_{0}=\frac{1}{8} \log n$. Then $r_{n} \geq 0.99$ holds for sufficiently large $n$.

Proof. Fix a set $C$ of $2 t$ pairs $x y(x \in X, y \in Y)$ forming a cycle, where $t \leq t_{0}$. We will estimate the probability $r_{n}(C)$ that $\mathbf{G}$ contains $C$ as a cycle. Let $\pi_{1}$, $\pi_{2}, \pi_{3}$ be bijections inducing $G=(V, E)$ and let $E_{i}$ be the set of edges induced by $\pi_{i}(i=1,2,3)$. Set $M_{i}=C \cap E_{i}$; clearly, $M_{i}$ is a matching. By [10, p. 129] we have $2^{2 t}+2$ ways to split $C$ into three matchings $M_{1}, M_{2}, M_{3}$. For each of them we have $a=\left(n-t_{1}\right) !\left(n-t_{2}\right) !\left(n-t_{3}\right) !$ triples $\pi_{1}, \pi_{2}, \pi_{3}$ with $E_{i} \cap C=$ $M_{i}(i=1,2,3)$, where $\left|M_{i}\right|=t_{i}(i=1,2,3)$.

Hence

$$
r_{n}(C) \leq\left(2^{2 t}+2\right) \cdot \operatorname{Max} \frac{\left(n-t_{1}\right) !\left(n-t_{2}\right) !\left(n-t_{3}\right) !}{(n !)^{3}} \leq \frac{2^{2 t+1}}{n^{2 t}}
$$

for sufficiently large $n$ (as $t_{i} \ll \sqrt{n}$ ). The number of cycles of length $2 t$ formed by pairs $x y, x \in X, y \in Y$, is

$$
\left(\begin{array}{l}
n \\
t
\end{array}\right)^{2} t !(t-1) ! \leq \frac{n^{2 t}}{t}
$$

Thus the expected number of cycles of length $\leq 2 t_{0}$ contained in $\mathbf{G}$ is at most

$$
\sum_{t \leq t_{0}} \frac{2^{2 t+1}}{t}<\frac{\sqrt{n}}{100}
$$


Hence we infer that the probability $\geq 0.99, \mathrm{G}$ contains at most $\sqrt{n}$ cycles of length $\leq 2 t_{0}$. Choosing an edge from each of these cycles we get the required set of edges.

Now we are ready to finish the proof of the lemma. Due to Claims 1-3, for sufficiently large $n$ there exists a bipartite graph $G=(V, E)$ on $2 n$ vertices that is 3-regular, has the partition property (ii), and only $\leq \sqrt{n} \cdot 2 t_{0}$ of its edges is contained in induced cycles of length $\leq 2 t_{0}$.

We modify $G$ to get a new graph satisfying (i)-(iii) of the lemma. Consider an edge $x y$ of $G$ that is contained in a cycle $C$ of length $\leq 2 t_{0}$. Choose another edge $x^{\prime} y^{\prime}$ such that the distance from $x$ to $x^{\prime}$ in $G$ is $\geq 2 t_{0}+1$ and that $x^{\prime} y^{\prime}$ is contained in no cycle of length $\leq 2 t_{0}$; such $x^{\prime} y^{\prime}$ does exist, for at most $\frac{1}{8} n \log n$ vertices are contained in cycles of length $\leq 2 t_{0}$ and at most $3\left(2^{2 t_{0}+1}-1\right) \cdot \sqrt{n}$ vertices $x^{\prime} \neq x$ are joined with $x$ by a path of length $\leq 2 t_{0}+1$. Delete edges $x y$, $x^{\prime} y^{\prime}$ from $G$ and add $x y^{\prime}, y x^{\prime}$ to $G$. This does destroy the cycle $C$ but does not create any new cycle of length $\leq 2 t_{0}$; moreover, the resulting graph remains 3-regular. Repeating this procedure at most $\sqrt{n}$ times (once for each short cycle) we get a 3-regular graph without cycles of length $\leq 2 t_{0}$. Also, the new graph satisfies property (ii) with $|M| \geq \frac{1}{33} n-\sqrt{n}$, i.e., $|M| \geq n / 34$ for sufficiently large $n$.

Lemma 2. The graph $G=(V, E)$ from Lemma 1 has the following property: for every function $f: V \rightarrow R$ there is an induced subtree $T$ of $G$ such that:

(i) $\operatorname{diam} T \leq 2 t_{0}$.

(ii) $|L| \geq n^{1 / 17}$, where $L$ is the set of leaves of $T$.

(iii) $f(x) \geq f(y)$ whenever $x$ is a leaf and $y$ is a nonleaf in $T$.

Proof. For every $x \in V$, let $L_{x}$ be the set of all vertices of $V$ where the distance from $x$ is $t_{1}=\left\lfloor t_{0} / 2\right\rfloor-1$. As $G$ does not contain cycles of length $\leq 2 t_{0}$ and $G$ is 3-regular, $L_{x}$ has cardinality

$$
S=3.2^{t_{1}-1}<\sqrt{n}
$$

Write $V$ as $V=\left\{v_{1}, \ldots, v_{2 n}\right\}$ where $f\left(v_{1}\right) \geq f\left(v_{2}\right) \geq \cdots \geq f\left(v_{2 n}\right)$. Choose $j$ such that for $P=\left\{v_{1}, \ldots, v_{j}\right\}$ and for $B=\bigcup_{x \in P} L_{x}$ we have

$$
n-\sqrt{n} \leq|B| \leq n
$$

and put $A=V-B$. Choose $A^{\prime} \subset A, B^{\prime} \supset B$ with $\left|A^{\prime}\right|=\left|B^{\prime}\right|=n$. By Lemma 1 , condition (ii), there exists a matching $M$ joining $A^{\prime}$ with $B^{\prime}$ with $|M| \geq \frac{1}{34} n$. Let $A_{1}$ be the set of vertices $x$ in $A^{\prime}$ such that $x y \in M$ for some $y \in B$. As $\left|B^{\prime}-B\right|<\sqrt{n}$ we have $A_{1} \geq \frac{1}{34} n-\sqrt{n}$ and for $A_{2}=A-A_{1}$ we have $\left|A_{2}\right|=|A|-\left|A_{1}\right| \leq \frac{33}{34} n+2 \sqrt{n} \leq$ $\frac{34}{35} n$ for sufficiently large $n$.

(a) We claim that there exists $x_{0} \in A$ such that

$$
\left|L_{x_{0}}-A_{2}\right| \geq \frac{S}{35} .
$$


Indeed, otherwise $\left|L_{x} \cap A_{2}\right|>\frac{34}{35} S$ for every $x \in A$. Denote by $N$ the number of pairs $(x, y)$ with $x \in A, y \in A_{2}$, and $y \in L_{x}$ (equivalently, $x \in L_{y}$ ). Then, on one hand,

$$
N=\sum_{x \in A}\left|L_{x} \cap A_{2}\right|>|A| \frac{34}{35} S \geq \frac{34}{35} n S
$$

and, on the other hand,

$$
N=\sum_{y \in A_{2}}\left|L_{y} \cap A \leq\right| A_{2} \mid S \leq \frac{34}{35} n S
$$

a contradiction.

(b) Let $L_{x_{0}}-A_{2}=\left\{x_{1}, \ldots, x_{m}\right\}, m \geq S / 35$. For every $i \in\{1, \ldots, m\}$, we have a path $P_{i}$ of length $t_{1}$ from $x_{0}$ to $x_{i}$ and a path $Q_{i}$ of length $\leq t_{1}+1$ from $x_{i}$ to some $z_{i} \in P$ (the existence of $Q_{i}$ follows by the definition of $B$ if $x_{i} \in B$; if $x_{i} \in A_{1}$ we use an $M$-edge $x_{i} y$ with $y \in B$ and a path of length $=t_{1}$ from $y$ to a vertex in $P$ ). As the $x_{i}$ 's are pairwise distinct and $G$ does not contain cycles of length $2 t_{0} \geq$ $4 t_{1}+2$, the $z_{1}$ 's are pairwise distinct, too, and the union of $P_{i}$ 's and $Q_{i}$ 's is an induced subtree $T$ of $G$. Assuming, in addition, that $z_{i}$ is the only $P$-vertex on $Q_{i}$, the tree $T$ satisfies (iii) due to the definition of $P$. We have diam $T \leq$ $4 t_{1}+2 \leq 2 t_{0}$ and $|L|=m \geq S / 35=\frac{3}{35} 2^{\left|t_{0} / 2\right|-2} \geq 2^{(1 / 17) \log n}=n^{1 / 17}$ for sufficiently large $n$.

Proof of the Theorem. Let $G=(V, E)$ be the 3-regular bipartite graph on $2 n$ vertices from Lemma 2 ; let $x \rightarrow \vec{x}(x \in V)$ be its representation in $R^{d}$ with $t=1$. Set $f(x)=\|\bar{x}\|$ for $x \in V$. Let $T$ be the induced subtree of Lemma 2. Thus we have $\gamma>0$ such that $\|\bar{x}\| \geq \gamma$ or $\|\bar{x}\| \leq \gamma$ if $x$ is a leaf or a nonleaf in $T$, respectively. Let $x_{0}$ be a nonleaf such that the distance from $x_{0}$ to each of the leaves is at most $t_{0}$. For every leaf $x$, let $f_{x}$ and $g_{x}$ be vertices on the path from $x_{0}$ to $x$ where the distance from $x$ is 1 and 2 , respectively.

Let $L_{1}$ be a maximal set of leaves with the property

$$
x, y \in L_{1}, \quad x \neq y \text { implies } g_{x} \neq g_{y} \text { and } g_{x} g_{y} \notin E
$$

If follows easily from the 3-regularity of $G$ and the maximality of $L_{1}$ that $\left|L_{1}\right| \geq|L| / 8 \geq n^{1 / 17} / 8$. For every $x \in L_{1}$ set $\tilde{x}=\alpha \bar{x}+(1-\alpha) \bar{g}_{x}$ where $\alpha \in\langle 0,1\rangle$ is chosen so that $\|\tilde{x}\|=\gamma$; such $\alpha$ does exist because $\|\tilde{x}\|=\|\bar{x}\| \geq \gamma$ for $\alpha=0$ and $\|\tilde{x}\|=\left\|\bar{g}_{x}\right\| \leq y$ for $\alpha=1$; for $x \in L_{1}$ set $\tilde{x}=\bar{x}$. Then for $x, y \in L_{1}, x \neq y$,

$$
\begin{aligned}
\tilde{x} \tilde{y} & =\left[\alpha \bar{x}+(1-\alpha) \bar{g}_{x}\right]\left[\beta \bar{y}+(1-\beta) \bar{g}_{y}\right]<1, \\
\tilde{x} \tilde{f}_{x} & =\left[\alpha \bar{x}+(1-\alpha) \bar{g}_{x}\right] \vec{f}_{x} \geq 1 .
\end{aligned}
$$

For every $x \in L_{1}$, let $K_{x}$ be the ball with center $\tilde{x}$ and radius $r=\sqrt{2 \gamma^{2}-2} / 2$. We have for $x, y \in L_{1}$

$$
\|\tilde{x}-\tilde{y}\|=\left(\tilde{x}^{2}-2 \tilde{x} \tilde{y}+\tilde{y}^{2}\right)^{1 / 2}>\left(2 \gamma^{2}-2\right)^{1 / 2}=2 r .
$$


Hence the balls $K_{x}$ are pairwise disjoint. On the other hand, if $x_{0}, x_{1}, \ldots, x_{t}=x$ is a path from $x_{0}$ to $x \in L$, then

$$
\left\|\tilde{x}_{i}-\tilde{x}_{i+1}\right\|=\left(\tilde{x}_{i}^{2}-2 \tilde{x}_{i} \tilde{x}_{i+1}+\tilde{x}_{i+1}^{2}\right)^{1 / 2} \leq\left(2 \gamma^{2}-2\right)^{1 / 2}=2 r
$$

hence balls $K_{x}$ are contained in the ball with center $x_{0}$ and radius $\leq\left(2 t_{0}+1\right) r$. Then

$$
\left|L_{1}\right| V_{d} r^{d} \leq V_{d}\left[\left(2 t_{0}+1\right) r\right]^{d}
$$

where $V_{d}$ is the volume of the unit ball in $R^{d}$. It follows

$$
n^{1 / 17} / 8 \leq\left(2 t_{0}+1\right)^{d}
$$

and thus

$$
d \geq \frac{\log \left(n^{1 / 17} / 8\right)}{\log \left(2 t_{0}+1\right)} \geq \frac{\frac{1}{17} \log n-3}{\log (\log n)-2} \geq \frac{\log n}{18 \log (\log n)}
$$

for sufficiently large $n$.

\section{References}

1. P. Frankl, H. Maehara, On the contact dimension of graphs, to appear.

2. P. Frankl, H. Maehara, The Johnson-Lindenstrauss lemma and the sphericity of some graphs, to appear.

3. H. Maehara, On the sphericity for the join of many graphs, Discrete Math. 49 (1984), 311-313.

4. H. Maehara, Space graphs and sphericity, Discrete Appl. Math. 7 (1984), 55-64.

5. H. E. Warren, Lower bounds of approximation by nonlinear manifolds.

6. V. Chvátal, P. L. Hammer, Aggregation of inequalities in integer programming, Ann. Discrete Math. 1 (1977), 145-162.

7. J. Reiterman, V. Rödl, E. Šinajová, Geometrical embeddings of graphs, Discrete Math., to appear.

8. G. P. Egoryčev, Solution of the van der Waerden Problem for Permanents, IFSO 13M, Akad. Nauk SSSR.

9. D. 1. Falikman, A proof of the van der Waerden conjecture on the permanent of a doubly stochastic matrix, Mat. Zametki 29 (1981), 931-938.

10. J. A. Bondy, U. S. R. Murty, Graph Theory with Applications, Macmillan, 1978.

Received December 8, 1986, and in revised form July 10, 1987. 